\title{
Endoscopic stenting versus surgical colostomy for the management of malignant colonic obstruction: comparison of hospital costs and clinical outcomes
}

\author{
Shyam Varadarajulu • Ann Roy • Tercio Lopes • \\ Ernesto R. Drelichman • Micheline Kim
}

Received: 25 September 2010/Accepted: 27 November 2010/Published online: 4 February 2011

(c) The Author(s) 2011. This article is published with open access at Springerlink.com

\begin{abstract}
Background Although stent placement is increasingly performed, colostomy still is considered the gold standard for emergent relief of malignant colonic obstruction (MCO). This study aimed to compare hospital costs and clinical outcomes between patients undergoing colostomy and those undergoing stenting for the management of MCO.

Methods A retrospective claims analysis of the Medicare Provider Analysis and Review (MedPAR) data set was conducted to identify inpatient hospitalizations for colostomy or stent placement for the treatment of colon cancer (2007-2008). The outcomes evaluated using MedPAR compared the total length of hospital stay (LOS) and the costs associated with both techniques. Because MedPAR is a claims data set that does not provide outcomes at a patient level, a single-institution retrospective case-control study was conducted in which each stent placement patient was matched with two colostomy patients during the same period. Outcome measures (institutional data) were used to compare rates of treatment success, postprocedure LOS, and reinterventions between the two cohorts.
\end{abstract}

Electronic supplementary material The online version of this article (doi:10.1007/s00464-010-1523-y) contains supplementary material, which is available to authorized users.

S. Varadarajulu $(\bowtie) \cdot$ T. Lopes · E. R. Drelichman

Basil I. Hirschowitz Endoscopic Center of Excellence,

University of Alabama at Birmingham, JT 664,

1530-3rd Avenue S, Birmingham, AL 35294-0012, USA

e-mail: svaradarajulu@yahoo.com

A. Roy · M. Kim

Health Economics and Reimbursement, Boston Scientific

Corporation, Natick, MA, USA
Results The MedPAR data evaluated 778 stent placements and 5,868 colostomy hospitalizations. There were no differences in gender, age distribution, or comorbidity between the two groups. Compared with colostomy, the median LOS ( 8 vs. 12 days; $p<0.0001$ ) and the median cost $(\$ 15,071$ vs. $\$ 24,695 ; p<0.001)$ per claim were significantly less for stent placement. Stent placement was more commonly performed at urban versus rural hospitals ( $84 \%$ vs. $16 \% ; p<0.0001)$, teaching versus nonteaching hospitals $(56 \%$ vs. $44 \% ; p=0.0058)$ and larger versus smaller institutions (mean bed capacity, 331 vs. 227; $p<0.0001)$. The institution data included 12 patients who underwent stent placement and 24 who underwent colostomy. Although both methods were technically successful, the median postprocedure LOS (2.17 vs. 10.58 days; $p=0.0004)$ and the rate of readmissions for complications $(0 \%$ vs. $25 \% ; p=0.01)$ were significantly lower for stent placement.

Conclusion Although the technical and clinical outcomes for colostomy and stent placement appear comparable, stent placement is less costly and associated with shorter LOS and fewer complications. Dissemination of stent placement beyond large teaching hospitals located in urban areas as a treatment for MCO is important given its implications for patient care and resource use.

Keywords Colonic stenting $\cdot$ Colostomy $\cdot$ Costs $\cdot$ Health resources

Approximately $8 \%$ to $29 \%$ of patients with colorectal cancer present with acute malignant colonic obstruction (MCO) [1]. Although surgical decompression is the standard of care, the rates for mortality (15-20\%) and morbidity $(45-50 \%)$ in this context are significantly higher 
than the mortality rate for elective surgery $(<6 \%)[2,3]$. In addition, curative resection is not possible for one-third of the patients presenting with acute MCO due to locally advanced disease, distant metastasis, or severe comorbidities [4].

Therefore, the traditional management approach is an emergency open surgery to relieve the obstruction and resect the tumor. Many patients, particularly those with obstructing left-sided colon cancer, end up with temporary or permanent stomas, which can adversely affect their health-related quality of life $[5,6]$.

Numerous studies have shown that self-expandable metal stent (SEMS) placement is a relatively simple and safe alternative to standard surgical management because it obviates the need for emergency surgery or colostomy $[7,8]$. In a pooled analysis of SEMS, the rates of technical success and clinical effectiveness for relief of acute MCO were reported to be greater than $90 \%$ [9]. The procedure is effective both for palliation in patients with inoperable disease and for temporary preoperative decompression (bridge to surgery) in surgical candidates [10].

In a randomized trial that included 48 patients with obstructive left-sided colon cancer, $67 \%$ of the patients who underwent endolaparoscopic surgery (colonic stenting followed by elective laparoscopic resection) had successful one-stage operations compared with only $38 \%$ of the patients who underwent emergent open surgery [11]. Nevertheless, a SEMS is expensive, and it is unclear whether its use is cost effective compared with colostomy. Conclusions from computer-assisted decision analyses and direct-cost European studies have been conflicting [12-17]. Because all clinical studies were from single centers and included a small cohort of patients, the general applicability of these data is debatable.

The objectives of the current study were to compare the hospital costs and hospital length of stay (LOS) at a national level by using a claims database and to compare the clinical outcomes at a patient level by conducting a single-institution retrospective case-control study for patients undergoing colostomy versus stenting for management of MCO.

\section{Materials and methods}

This study was executed in two parts. First, MedPAR data was used to evaluate hospital costs and the LOS for patients who underwent colostomy or stenting for MCO. Second, because clinical outcomes, except for LOS, cannot be evaluated using MedPAR, a retrospective case-control study was conducted to evaluate patient outcomes at an institutional level.
MedPAR data source

A retrospective analysis was conducted using the 2007-2008 MedPAR data. The MedPAR database contains complete inpatient hospitalization records for the entire U.S. Medicare population, or 36.8 million covered lives. These claims report patient demographic information such as age, sex, diagnosis, and comorbidities. In addition, information regarding the hospitalization is provided including LOS, diagnostic testing, therapeutic procedures, and the charge for each hospitalization. The MedPAR data set also provides geographic and demographic information for the hospitals in which those claims were generated including location, associations, teaching status, number of beds, and number of full-time employees.

\section{Patient population}

The study population consisted of all unique hospitalization claims for the placement of a colonic stent or for a colostomy procedure. Claims were included based on primary diagnosis and procedure. In 2007 and 2008, no unique International Classification of Diseases (ICD-9$\mathrm{CM})$ code was in place for reporting colonic stent placement. As such, the following proxy was developed to identify claims: a colorectal cancer diagnosis with any one of the following ICD-9-CM diagnosis codes: 153.0-153.3, 153.6-154.1 reported with any one of the following endoscopy ICD-9-CM codes: 45.22, 46.79, 46.85 reported with revenue code 0278: medical/surgical supplies other implant. Colostomy claims were identified by a colorectal cancer diagnosis reported with any one of the following surgical resection ICD-9-CM codes: 45.73-45.80 reported with any one of the following colostomy ICD-9-CM codes: 46.03, 46.10-46.11, 46.13. Claims that had both stent placement and colostomy reported together were excluded from the analysis.

\section{Outcome measures}

The outcomes evaluated compared the total hospitalization costs and the total LOS associated with each technique. Using the Medicare Cost Report, the hospitalization costs were derived by applying the cost-to-charge ratio to the reported charges in MedPAR. A secondary analysis also was conducted to analyze the demographics of the hospitals in which the procedures were performed.

Institutional data

Because MedPAR is a claims database that does not provide outcomes at a patient level, a single-institution retrospective case-control study was conducted in which each 
patient who underwent a stent placement was matched with two colostomy patients during the same period (2007-2008).

\section{Patient population}

A retrospective analysis was conducted with consecutive patients ( $>19$ years of age) who underwent surgical diversion or colorectal stenting for management of acute MCO. Patients were identified from the endoscopy and surgery databases. The inclusion criteria specified patients older than 19 years with an underlying diagnosis of colorectal cancer who had undergone procedures for relief of acute obstruction. The exclusion criteria ruled out patients who had undergone colostomy or stenting for benign diseases or colostomy for perforated colon cancer.

For each patient treated with colonic stents, two patients who underwent emergency surgery (cancer resection with colostomy) for acute MCO were matched by an independent observer for the following variables: location of cancer, cancer stage, and presence of distant metastasis. The independent observer who conducted the matching process was blinded to all clinical outcomes and costs. The medical records of all the study subjects were reviewed for patient demographics, clinical presentation, comorbidities, laboratory investigations, site of large bowel obstruction, cancer staging, and radiologic investigations.

\section{Colonic stenting}

Each SEMS (Ultraflex Precision; Boston Scientific Corp, Natick, MA, USA) was deployed under fluoroscopic guidance with the patient in the left lateral position using a combination of intravenous midazolam and meperidine (Video 1). The stents measured $25 \mathrm{~mm}$ in the body and $30 \mathrm{~mm}$ in the proximal flare and were 6,9 , or $12 \mathrm{~cm}$ in length.

At colonoscopy, a 0.035-in. guidewire was first advanced across the stricture. A 5-Fr endoscopic retrograde cholangiopancreatography (ERCP) cannula then was advanced over the guidewire, and contrast was injected to assess the length of the stricture. The SEMS delivery system then was advanced over the guidewire, and after satisfactory positioning of the delivery catheter had been confirmed by fluoroscopy, the SEMS was deployed.

\section{Surgical resection and colostomy (Hartman's procedure)}

Briefly, after a midline incision had been performed, the obstructive tumor was resected and the proximal colon externalized as a colostomy. The rectal stump was sealed with surgical staples during the resection.

\section{Outcome measures}

The rates for treatment success, complications, and reinterventions were compared as well as the length of the postprocedure hospital stay between each treatment method.

\section{Consent}

All the patients provided informed consent for undergoing the procedures, and the study was approved by the University of Alabama at Birmingham Institutional Review Board.

Statistical analysis

\section{MedPAR data}

All analyses were performed using SAS version 9.2 (SAS Institute, Cary, NC, USA). Discrete data are reported as frequencies and continuous data as median and mean. Using a chi-square test, the patient demographics and comorbidities reported on the claims were compared across the stenting and colostomy cohorts. Covariates were not adjusted for in the analysis of health resources due to constraints of using MedPAR data.

\section{Institutional data}

Statistical analysis was performed using Stata 9.2 (StataCorp LP, College Station, TX, USA). Patient demographics and disease characteristics were compared across the two groups (SEMS and surgery). A two-sample $t$-test was used to compare the means of the continuous variables such as age, albumin levels, and Charlson's score. A chi-square test was used to compare the proportions of disease characteristics (Table 1) and outcome measures (Table 2) across the two groups.

\section{Results}

\section{MedPAR data}

A total of 778 colonic stent placements and 5,868 colostomy claims met the criteria for inclusion in the study. The age, gender, and comorbidities of the reported patients in both cohorts are shown in Table 3. The colostomy cohort had more reported women than the stenting cohort. Except for liver disease and diabetes, the two cohorts did not differ significantly in terms of comorbid conditions. 
Table 1 Patient demographics and disease characteristics at presentation (institutional data)

\begin{tabular}{llll}
\hline & $\begin{array}{l}\text { Stenting } \\
\text { cohort } \\
(n=12)\end{array}$ & $\begin{array}{l}\text { Colostomy } \\
\text { cohort } \\
(n=24)\end{array}$ & $p$ Value \\
\hline Mean age (year) & 67.08 & 58.25 & 0.052 \\
Gender (\% female) & 50 & 54.1 & 0.813 \\
Race (\% African American) & 33.33 & 45.83 & 0.469 \\
Cancer site: $n(\%)$ & & & 1.000 \\
Sigmoid & $9(75)$ & $18(75)$ & \\
Rectum & $2(17)$ & $4(17)$ & \\
Transverse & $1(8)$ & $2(8)$ & \\
Histology: $n(\%)$ (adenocarcinoma) & $12(100)$ & $24(100)$ & 1.000 \\
Stage: $n(\%)$ & & & 0.655 \\
2 & $2(17)$ & $4(17)$ & \\
3 & $1(8)$ & $5(21)$ & \\
4 & $9(75)$ & $15(62)$ & \\
Metastasis: $n$ (\%) & $10(83)$ & $15(63)$ & 0.186 \\
Mean albumin (mg/dl) & 3.10 & 2.69 & 0.212 \\
Mean Charlson's score & 6.08 & 5.54 & 0.426 \\
\hline
\end{tabular}

\section{Health resource use}

The median aggregate hospital days or the total LOS from admission to discharge was 8 days for colonic stent placement claims and 12 days for colostomy claims $(p<0.0001)$. The stent placement claims had significantly lower total median hospital costs per claim than the colostomy claims. The mean hospital costs for the stent placement cohort were $\$ 21,771$ versus $\$ 33,383$ for the colostomy cohort $(p<0.0001)$.

Stent placement was more commonly performed at urban $(84 \%)$ versus rural hospitals $(16 \%)(p<0.0001)$ and at teaching $(56 \%)$ versus nonteaching $(44 \%)$ institutions $(p<0.006)$. In addition, stent placement was more commonly performed at larger institutions with a mean capacity of 331 beds than at smaller institutions with a mean capacity of 227 beds for colostomy procedures $(p<0.0001)$.
Institutional data

The single-institution retrospective case-control study involved 12 patients who underwent colonic stenting and 24 patients who underwent surgery for acute MCO. Two SEMS patients were excluded because they returned to the referring facility for further care after stent placement. Neither of these excluded patients experienced any intraprocedural complications.

After each SEMS patient had been matched with two patients who had undergone surgery, the study cohort comprised of a total of 36 patients. Table 1 displays the demographics and disease characteristics of all the patients at presentation. The two cohorts did not differ significantly in terms of patient demographics, tumor characteristics, or comorbidities.

Colostomy and SEMS placements were successful in relieving the obstruction in all the patients. Whereas the mean postprocedure LOS was significantly shorter $(2.17$ vs. 10.58 days; $p=0.004$ ), there was no significant difference in the preprocedure LOS between the patients who underwent SEMS placements (1 day) and those that had colostomy (1.4 days) $(p=0.72)$.

One SEMS patient experienced self-limited lower gastrointestinal bleeding during the initial hospitalization that did not require reintervention or blood transfusion, whereas eight patients $(33 \%)$ in the surgery group experienced complications ( $8.3 \%$ vs. $33.3 \% ; p=0.08$ ) including sepsis $(n=1)$, parastomal hernia $(n=1)$, anastomotic leak $(n=1)$, peristomal abscess $(n=1)$, intraabdominal abscess $(n=1)$, small bowel obstruction $(n=1)$, enterocutaneous fistula $(n=1)$, and pelvic abscess $(n=1)$.

Whereas none of the patients who underwent SEMS placement required a readmission for stent-related complications, six patients who underwent surgery required readmission for complications $(0 \%$ vs. $25 \%, p=0.019)$. One patient $(4 \%)$ in the surgical cohort died of sepsis 28 days after undergoing colostomy.

Of the 12 patients who underwent SEMS placements, 6 had elective one-stage operations for tumor resection. The remaining six 6 patients were treated palliatively. At the
Table 2 Clinical outcomes of SEMS versus surgery (institutional data)

SEMS self-expandable metal stent

\begin{tabular}{llll}
\hline & $\begin{array}{l}\text { Stenting cohort } \\
\left(\begin{array}{l}n=12) \\
n(\%)\end{array}\right.\end{array}$ & $\begin{array}{l}\text { Colostomy cohort } \\
(n=24) \\
n(\%)\end{array}$ & $p$ Value \\
\hline Technical success & $12(100)$ & $24(100)$ & 1.000 \\
Clinical success & $12(100)$ & $24(100)$ & 1.000 \\
Intraprocedure complication & $0(0)$ & $0(0)$ & 1.000 \\
Delayed complication & $1(8.3)$ & $8(33.3)$ & 0.081 \\
Readmittance for complication & $0(0)$ & $6(25)$ & 0.019 \\
Mean postprocedure hospital stay (days) & 2.17 & 10.58 & 0.004 \\
\hline
\end{tabular}


Table 3 Baseline demographics and co-morbid conditions of the stent placement and colostomy patient populations as reported in MedPAR claims (MedPAR data)

\begin{tabular}{|c|c|c|c|}
\hline & $\begin{array}{l}\text { Stenting } \\
\text { cohort } \\
(n=778) \\
\%\end{array}$ & $\begin{array}{l}\text { Colostomy } \\
\text { cohort } \\
(n=5,868) \\
\%\end{array}$ & $p$ Value \\
\hline \multicolumn{4}{|l|}{ Age group (years) } \\
\hline $25-44$ & 1.0 & 0.3 & 0.001 \\
\hline $45-64$ & 8.4 & 6.6 & 0.061 \\
\hline $65-69$ & 17.5 & 16.0 & 0.304 \\
\hline $70-74$ & 19.4 & 16.5 & 0.042 \\
\hline $75-79$ & 17.9 & 19.2 & 0.359 \\
\hline $80-84$ & 15.8 & 19.0 & 0.030 \\
\hline $85-89$ & 10.2 & 13.8 & 0.005 \\
\hline$>89$ & 9.8 & 8.6 & 0.266 \\
\hline Female & 45.6 & 52.2 & 0.001 \\
\hline \multicolumn{4}{|l|}{ Comorbid conditions } \\
\hline $\mathrm{CHF}$ & 12.3 & 12.8 & 0.738 \\
\hline COPD & 13.9 & 14.6 & 0.574 \\
\hline Cerebrovascular disease & 2.8 & 3.0 & 0.852 \\
\hline Diabetes without complications & 13.0 & 12.4 & 0.619 \\
\hline Diabetes with complications & 0.3 & 1.0 & 0.034 \\
\hline Myocardial infarction & 4.6 & 4.6 & 0.991 \\
\hline Moderate/severe liver disease & 1.9 & 0.3 & $<0.0001$ \\
\hline Peripheral vascular disease & 3.6 & 3.0 & 0.362 \\
\hline Renal disease & 7.3 & 5.7 & 0.075 \\
\hline
\end{tabular}

CHF congestive heart failure, $C O P D$ chronic obstructive pulmonary disease

last follow-up visit, only two of six patients who underwent elective resections were alive. The others had died of metastatic cancer or some other comorbidity. Of the six patients who underwent palliative stenting, follow-up data were available for four who died without requiring any reinterventions. The two remaining patients had died but were lost to follow-up evaluation after stent placement.

Of the 24 patients who had colostomy, 5 underwent surgical reanastomosis and were alive at a median followup time of 29 months (range, 13-46 months). Of the remaining 19 patients, 14 had died, 3 were receiving palliative therapy, and 2 were lost to follow-up evaluation.

\section{Discussion}

Colonic stenting is an effective alternative to surgery for both palliation and conversion of an emergent operation to an elective surgery. Three decision analyses and two case series, mostly from Canada and Europe, have attempted to address the cost effectiveness of the colonic stent approach [12-16]. In one decision analysis, colonic stenting was found to be more expensive than diverting colostomy, and in two others, stent placement was less costly than surgery, whether performed as resection with colostomy or as primary resection with anastomosis [12-14].

In the two case series, colon stenting was found to be more expensive than colostomy in the one series [15] and less expensive than surgical resection in the other [16]. The reason for this may be that diverting loop colostomy is performed as a palliative procedure through a smaller incision and is not as extensive an operation as resection and end colostomy. Although modeling is useful for comparison of costs and outcomes between different methods, the results are not expected to be precise because predictions are dependent on the accuracy of clinical outcome probabilities, many of which are unknown. Thus, the diverging results achieved by the aforementioned studies are not surprising.

In the current study, the median cost per claim was $\$ 9,600$ lower for patients undergoing SEMS placement. These findings involving 778 SEMS and 5,868 surgical claims are more realistic for the costs involving both treatment methods in the United States' population.

In a metaanalysis of 10 studies, the LOS was shorter by 8 days for the patients who underwent colon stenting than for those who underwent surgery [17]. However, this finding was associated with significant heterogeneity. In the current study, the median LOS per claim was shorter by 4 days for the patients who underwent stenting than for the surgical cohort. Whereas the total LOS can be estimated using the MedPAR database, it is not possible to assess the postprocedure LOS. When these clinical outcomes were evaluated at an institutional level, the postprocedure LOS was shorter by 8 days for patients who underwent colonic stenting.

Whereas 6 of 12 patients in the SEMS cohort underwent successful one-stage elective surgery, only 5 of 24 patients in the surgical cohort underwent reanastomosis at a later time. This is in line with the findings of a recent randomized trial in which a majority of patients who underwent emergency surgery for acute MCO had a permanent stoma [11]. Whereas no significant complication occurred in the SEMS group, eight patients in the surgery group experienced complications, and six patients required a readmission. Even in the absence of complications, surgical patients required lengthier postprocedure hospital stays for pain management, return of bowel function, and tolerance of regular diet.

Our study had some limitations. Although it would have been ideal to conduct a longitudinal study evaluating the two cohorts from the initial procedure to death, tracking procedures performed and associated costs, one limitation of the MedPAR claims database is the inability to run a longitudinal analysis. There are no unique patient 
identifiers to track the same patient over time. The MedPAR database allows only for cross-sectional studies to be performed.

In addition, outcomes data, such as procedural success and complications rates, cannot be evaluated using a claims database. It also is possible that some of the patients who underwent colostomy had failed attempts at prior stent placement. At an institutional level, because patients were not followed prospectively, it is likely that some minor adverse events were not captured.

Despite rigorous matching, it is possible that outcomes were influenced by the patient composition in each group. However, this may not be a major limitation because patients undergoing SEMS were older (67 vs. 58 years; $p=0.052$ ) and had marginally higher Charlson's scores (6.08 vs. $5.54 ; p=0.426$ ). With the exception of one patient who underwent stenting of the transverse colon, obstruction was limited to the left colon or rectum in all the other patients. Moreover, every attempt was made to maintain uniformity by matching both groups for site of obstruction, cancer stage, and presence of distant metastasis. Also, the Charlson's comorbidity scores were comparable between the two groups.

In addition, this case-control study examined a small sample of patients from a single institution. As a result, the ability to generalize these findings to a national sample is limited and is not the intent of this study. Because it was a retrospective case-control study, we could not present data on the rates of technical failure in the SEMS cohort or on the number of patients who failed stent placement and subsequently underwent surgery.

Analysis of the claims data showed that despite its inherent clinical advantages and cost savings, colonic stenting is more commonly performed at large urban teaching hospitals. We speculate that in large teaching institutions, better collaboration between the disciplines of medical gastroenterology and general surgery result in more patients undergoing SEMS placement in the emergent setting. In smaller and rural hospitals, patients probably are managed by the manner in which they are triaged at admission. Those admitted to the gastroenterology service are more likely to undergo stent placement, and those admitted to the surgical service are more likely to undergo resection.

It is our opinion that at least for patients with multiple comorbidity and poor functional status, colon stent placement should be the favored initial treatment approach because these patients are poor operative candidates and unlikely to endure a second surgery for reanastomosis. More education and training is needed to propagate the appropriate use of stents in patients presenting with acute MCO. Training in stent deployment should be incorporated in general surgery residency programs by the use of simulators or models, or by enabling residents to rotate in the medical gastroenterology service.

In conclusion, although the technical and clinical outcomes of colostomy and stent placement appear comparable in relieving obstruction, stent placement is less costly and associated with shorter LOS and fewer complications. Dissemination of stent placement beyond large teaching hospitals located in urban areas as a treatment for MCO is important given its implications for patient care and resource use.

Disclosures Shyam Varadarajulu and Ernesto R. Drelichman are consultants for Boston Scientific Corporation. Ann Roy and Micheline Kim are employees of Boston Scientific Corporation. Tercio Lopes has no conflicts of interest or financial ties to disclose.

Open Access This article is distributed under the terms of the Creative Commons Attribution Noncommercial License which permits any noncommercial use, distribution, and reproduction in any medium, provided the original author(s) and source are credited.

\section{References}

1. Deans GT, Krukowski AH, Irwin ST (1994) Malignant obstruction of the left colon. Br J Surg 81:1270-1276

2. Mulcahy HE, Skelly MM, Husain A, O'Donoghue DP (1996) Long-term outcome following curative surgery for malignant large bowel obstruction. Br J Surg 83:46-50

3. Griffith RS (1992) Preoperative evaluation: medical obstacles to surgery. Cancer 70:1333-1341

4. Wong RW, Rappaport WD, Witzke DW, Putnam CW, Hunter GC (1994) Factors influencing the safety of colostomy closure in the elderly. J Surg Res 57:289-292

5. Sprangers MA, Taal BG, Aaronson NK, te Velde A (1995) Quality of life in colorectal cancer: stoma vs nonstoma patients. Dis Colon Rectum 38:361-369

6. Dominitz JA, Provenzale D (1997) Patient preferences and quality of life associated with colorectal cancer screening. Am J Gastroenterol 92:2171-2178

7. Khot UP, Lang AW, Murali K, Parker MC (2002) Systematic review of the efficacy and safety of colorectal stents. Br J Surg 89:1096-1102

8. Watt AM, Faragher IG, Griffin TT, Rieger NA, Maddern GJ (2007) Self-expandable metallic stents for relieving malignant colorectal obstruction: a systematic review. Ann Surg 246:24-30

9. Sebastian S, Johnston S, Geoghegan T, Torreggiani W, Buckley M (2004) Pooled analysis of the efficacy and safety of selfexpanding metal stenting in malignant colorectal obstruction. Am J Gastroenterol 99:2051-2057

10. Tierney W, Chuttani R, Croffie J, DiSario J, Liu J, Mishkin DS, Shah R, Somogyi L, Petersen BT (2006) Enteral stents. Gastrointest Endosc 63:920-926

11. Cheung HYS, Chung CC, Tsang WWC, Wong JCH, Yau KKK, Li MKW (2009) Endolaparoscopic approach vs conventional open surgery in the treatment of obstructing left-sided colon cancer. Arch Surg 144:1127-1132

12. Singh H, Latosinsky S, Spiegel BM, Targownik LE (2006) The cost effectiveness of colonic stenting as a bridge to curative surgery in patients with acute left-sided malignant colonic 
obstruction: a Canadian perspective. Can J Gastroenterol 20:779-785

13. Govindarajan A, Naimark D, Coburn NG, Smith AJ, Law CHL (2007) Use of colonic stents in emergent malignant left colonic obstruction: a Markov chain Monte Carlo decision analysis. Dis Colon Rectum 50:1811-1824

14. Targownik LE, Spiegel BM, Sack J, Hines OJ, Dulai GS, Gralnek IM, Farrell JJ (2004) Colonic stent vs. emergency surgery for management of acute left-sided malignant colonic obstruction: a decision analysis. Gastrointest Endosc 60:865-874

15. Xinopoulos D, Dimitroulopoulos D, Theodosopoulos T, Tsamakidis K, Bitsakou G, Plataniotis G, Gontikakis M, Kontis M, Paraskevas I, Vassilobpoulos P, Paraskevas E (2004) Stenting or stoma creation for patients with inoperable malignant colonic obstructions? Results of a study and cost-effectiveness analysis. Surg Endosc 18:421-426

16. Binkert CA, Ledermann H, Jost R, Saurenmann P, Decurtins M, Zollikofer CL (1998) Acute colonic obstruction: clinical aspects and cost effectiveness of preoperative and palliative treatment with self-expanding metallic stents: a preliminary report. Radiology 206:199-204

17. Tilney HS, Lovegrove RE, Purkayastha S, Sains PS, WestonPetrides GK, Darzi AW, Tekkis PP, Heriot AG (2007) Comparison of colonic stenting and open surgery for malignant large bowel obstruction. Surg Endosc 21:225-233 